\title{
Observatory geoelectric fields induced in a two-layer lithosphere during magnetic storms
}

\author{
Jeffrey J Love ${ }^{1 *}$ and Andrei Swidinsky ${ }^{2}$
}

\begin{abstract}
We report on the development and validation of an algorithm for estimating geoelectric fields induced in the lithosphere beneath an observatory during a magnetic storm. To accommodate induction in three-dimensional lithospheric electrical conductivity, we analyze a simple nine-parameter model: two horizontal layers, each with uniform electrical conductivity properties given by independent distortion tensors. With Laplace transformation of the induction equations into the complex frequency domain, we obtain a transfer function describing induction of observatory geoelectric fields having frequency-dependent polarization. Upon inverse transformation back to the time domain, the convolution of the corresponding impulse-response function with a geomagnetic time series yields an estimated geoelectric time series. We obtain an optimized set of conductivity parameters using 1-s resolution geomagnetic and geoelectric field data collected at the Kakioka, Japan, observatory for five different intense magnetic storms, including the October 2003 Halloween storm; our estimated geoelectric field accounts for $93 \%$ of that measured during the Halloween storm. This work demonstrates the need for detailed modeling of the Earth's lithospheric conductivity structure and the utility of co-located geomagnetic and geoelectric monitoring.
\end{abstract}

Keywords: Magnetic storm; Induction hazard; Geomagnetic and geoelectric fields; Time-series analysis; Operational algorithm

\section{Introduction}

Magnetic storms are potentially hazardous for the activities and technological infrastructure of modern civilization (e.g., Baker et al. 2008; Cannon et al. 2013; Daglis 2004). Of particular concern is the storm-time induction of geoelectric fields in the Earth's lithosphere. Corresponding voltage differentials can find their way into electric-power grids through ground connections, driving uncontrolled electric currents that interfere with grid operation, damaging transformers, tripping protective relays, and sometimes causing blackouts (e.g., Boteler et al. 1998; Kappenman 2012; Thomson et al. 2010). The reality of this complicated chain of causes and effects was demonstrated during the great magnetic storm of March 1989 (Allen et al. 1989), which caused the complete collapse of the Hydro-Québec electric-power grid in Canada, temporarily leaving nine million people without electricity (Bolduc 2002). The possible occurrence in the future of an

\footnotetext{
*Correspondence: jlove@usgs.gov

1 US Geological Survey, Geomagnetism Program, Box 25046 MS 966 DFC, Denver, CO 80225, USA

Full list of author information is available at the end of the article
}

even more intense magnetic storm, one that could cause widespread loss of electric power, has motivated regulatory agencies to issue operational standards intended to mitigate the effects of induction hazards. Concerns across the private sector have motivated reinsurance companies to commission related assessments of risk (e.g., Lloyds 2013; Riswadkar and Dobbins 2010).

In this broad context of practical concerns, the evaluation of induction hazards (e.g., Love et al. 2014; Pulkkinen et al. 2012; Viljanen 1997; Wei et al. 2013) relies on geomagnetic field data collected at ground-based observatories (e.g., Love 2008; Macmillan 2007), facilities operated around the world (e.g., Love and Chulliat 2013; Love and Finn 2011; Minamoto 2013), as parts of national geophysical projects for geomagnetic field mapping and space weather monitoring. Many magnetic observatories have been in continuous operation for decades, and a few have been in operation for over a century. The geomagnetic history recorded in observatory data is useful for analysis of evolving solar-terrestrial interaction and extreme space weather events. Magnetic observatory data are also used as input for the development and testing of algorithms

\section{黑 Springer}

(c) 2015 Love and Swidinsky; licensee Springer. This is an Open Access article distributed under the terms of the Creative

Commons Attribution License (http://creativecommons.org/licenses/by/4.0), which permits unrestricted use, distribution, and reproduction in any medium, provided the original work is properly credited. 
that might be implemented in an operational setting for real-time evaluation of magnetic storm induction hazards.

In contrast, geoelectric field data are usually collected on a campaign basis at multiple stations deployed temporarily as parts of magnetotelluric (e.g., Chave and Jones 2012; Simpson and Bahr 2005) projects undertaken to infer localized lithospheric electrical conductivity (e.g., Schultz 2009; Uyeshima 2007); . More unusual are projects dedicated to the long-term collection of geoelectric data at geographically fixed stations. Such a project has, however, been maintained at the observatories of the Japan Meteorological Agency (JMA) for many years (Minamoto 2013), thus providing an opportunity to study the details of magnetic storm induction. We choose to analyze 1-s resolution magnetic and electric field data that were collected at JMA's Kakioka observatory and which record intense magnetic storms.

A magnetic storm is seen in observatory data as a temporary enhancement in geomagnetic and geoelectric disturbance, often commencing suddenly, followed by a day or two of growth and evolution, and, finally, a gradual return to a state of relative quiescence. Such nonstationary variation can be described, in terms of mathematics, as a superposition of transient exponential moment functions defined with initial conditions. This is the basis of Laplace transformation from the time domain to the complex frequency domain. Laplace transformation conveniently reduces differential equations in time to algebraic equations in frequency, and time-domain convolution is reduced to simple multiplication. With a parameterized model of the electrically conducting lithosphere, one can obtain a transfer function of complex frequency that describes the physical relationship between transient geomagnetic variation and the resulting induced geoelectric field. Inverse Laplace transformation gives a time-domain impulse-response function that, when convolved with a time series recording geomagnetic variation, gives an estimate of the geoelectric field that is time-causal, a quality of importance for real-time operational algorithms.

Love and Swidinsky (2014) used a simple fourparameter model of lithospheric conductivity: a uniform half-space with a galvanic distortion tensor used to accommodate localized geoelectric polarization arising from regional three-dimensional conductivity. They showed that the corresponding impulse-response function could account for $87 \%$ of the geoelectric variation induced at Kakioka during the Halloween magnetic storm of October 2003, but the unmodeled residual 13\% includes high-frequency (sub-minute) geoelectric variation that might be of importance for hazard assessment and mitigation. Following on from the work of Love and Swidinsky, and guided by geophysical and geological concepts, we have a practical goal: improved algorithmic estimation of storm-time geoelectric variation. We analyze the predictive utility of a two-layer model of lithospheric conductivity; with each layer parameterized by a separate distortion tensor, the corresponding transfer function can accommodate frequency dependence in geoelectric polarization. We use the nine-parameter time-domain impulse-response function to estimate geoelectric variation recorded in data from Kakioka during five different magnetic storms.

\section{Observatory data}

We analyze 1-s magnetic data time series from the Kakioka observatory (geographic coordinates: $36.23 \mathrm{~N}$, 140.19 E). Reported by the JMA since 1983, these data were collected using a fluxgate sensor. Each datum is an instantaneous 'spot' measurement of the natural geomagnetic field variation. The magnetic field data have been calibrated for drift in sensor-system response. As a time series, the 1-s data are sufficient to resolve most of the time dependence of rapid and impulsive magnetic variation realized during magnetic storms. Measured vector values can be represented as a discrete sequence, $\mathbf{B}^{m}\left(t_{i}\right)$, for time-stamp values $t_{i}, t_{i+1}, t_{i+2}, \cdots$, where $\tau=t_{i+1}-t_{i}$ is the constant 1-s sampling interval. These are reported in terms of geographic-polar components $\left(B_{h}\right.$ horizontal intensity; $B_{d}^{m}$ declination; $B_{z}^{m}$ down). We convert the horizontal components to geographic-Cartesian components, ( $B_{x}^{m}$ north; $B_{y}^{m}$ east).

We also analyze 1-s electric data time series from the Kakioka observatory; 1-s resolution electric data have been measured at Kakioka and reported since 2000. These were collected by measuring the potential difference between pairs of grounded electrodes, oriented along north-south and east-west lines and separated by $190 \mathrm{~m}$. Measured electric vector values are reported in geographic-Cartesian vector components $\mathbf{E}^{m}=\left(E_{x}^{m}\right.$ north; $E_{y}^{m}$ east $)$; no measurements were made of the geoelectric vertical component. The electric field data contain a slow drift in baseline that is possibly an artifact of changes in electrode grounding, a common problem (e.g., Ferguson 2012); this part of the time series is not relevant to our analysis, and so, we subtract a trend line from each vector component. Both the magnetic and electric 1-s data are very complete in time for the durations we analyze; we fill a few gaps and replace a few locked values (consecutive values that are evidently not changing in time) by linear interpolation.

\section{Five intense magnetic storms}

We analyze data durations recording five intense magnetic storms, each with its own distinctive time-series variation; see Table 1. In Figure 1a, we show the measured $\mathbf{B}^{m}=\left(B_{x}^{m}\right.$ north, blue; $B_{y}^{m}$ east, green $)$ geomagnetic time series for the Halloween storm of 29 to 31 October 2003 (Balch et al. 2004, Gopalswamy et al. 2005). 
Table 1 Summary of magnetic storm and model properties

\begin{tabular}{|c|c|c|c|c|c|c|c|c|c|c|c|c|c|c|c|c|c|c|c|c|c|c|}
\hline \multirow[b]{2}{*}{ \# } & \multirow[b]{2}{*}{ Name } & \multicolumn{2}{|c|}{ Start day } & \multicolumn{2}{|c|}{ End day } & \multirow[b]{2}{*}{ yyyy } & \multirow{2}{*}{$\begin{array}{c}\text { Max } \\
-D s t \\
n T\end{array}$} & \multirow{2}{*}{$\begin{array}{c}\text { Max } \\
\left|\Delta B_{x}\right| \\
\mathrm{nT} / \mathrm{s}\end{array}$} & \multirow{2}{*}{$\begin{array}{c}\text { Ave } \\
\left|\Delta B_{x}\right| \\
\mathrm{nT} / \mathrm{s}\end{array}$} & \multicolumn{6}{|c|}{ Top } & \multicolumn{5}{|c|}{ Half } & \multicolumn{2}{|c|}{ Misfit $\varepsilon^{2}$} \\
\hline & & dd & $\mathrm{mm}$ & dd & $\mathrm{mm}$ & & & & & $G_{x x}$ & $G_{x y}$ & $G_{y x}$ & $G_{y y}$ & $\begin{array}{c}1 / a_{T} \\
\mathrm{~S}\end{array}$ & $\begin{array}{l}b_{T} \\
\mathrm{~km}\end{array}$ & $G_{x x}$ & $G_{x y}$ & $G_{y x}$ & $G_{y y}$ & $\begin{array}{r}\sigma_{H} \\
\mathrm{mS} / \mathrm{m}\end{array}$ & Opt & A \\
\hline 1 & Bastille & 15 & 07 & 16 & 07 & 2000 & 301 & 4.57 & 0.06 & -0.01 & 0.12 & -0.20 & 1.39 & 35.73 & 43.71 & 0.07 & 0.22 & -0.05 & 1.39 & 0.32 & 0.0668 & 0.0779 \\
\hline 2 & & 30 & 03 & 01 & 04 & 2001 & 387 & 1.62 & 0.03 & -0.08 & -0.01 & -0.82 & 1.15 & 17.41 & 49.53 & 0.05 & 0.15 & -0.33 & 1.37 & 0.38 & 0.0530 & 0.0549 \\
\hline 3 & & 05 & 11 & 07 & 11 & 2001 & 292 & 5.88 & 0.02 & 0.07 & -0.03 & -0.56 & 1.30 & 10.80 & 47.25 & 0.08 & 0.16 & -0.21 & 1.39 & 0.43 & 0.0449 & 0.0540 \\
\hline 4 & Halloween & 29 & 10 & 31 & 10 & 2003 & 383 & 5.58 & 0.07 & -0.09 & 0.04 & -1.08 & 0.91 & 47.77 & 49.83 & 0.03 & 0.18 & -0.58 & 1.28 & 0.32 & 0.0686 & 0.0816 \\
\hline 5 & & 20 & 11 & 21 & 11 & 2003 & 422 & 1.12 & 0.03 & -0.02 & 0.00 & -0.69 & 1.24 & 25.21 & 54.07 & 0.08 & 0.17 & -0.22 & 1.38 & 0.29 & 0.0540 & 0.0610 \\
\hline A & Average & & & & & & & & & -0.03 & 0.02 & -0.70 & 1.23 & 24.08 & 47.50 & 0.06 & 0.18 & -0.28 & 1.37 & 0.35 & & \\
\hline
\end{tabular}



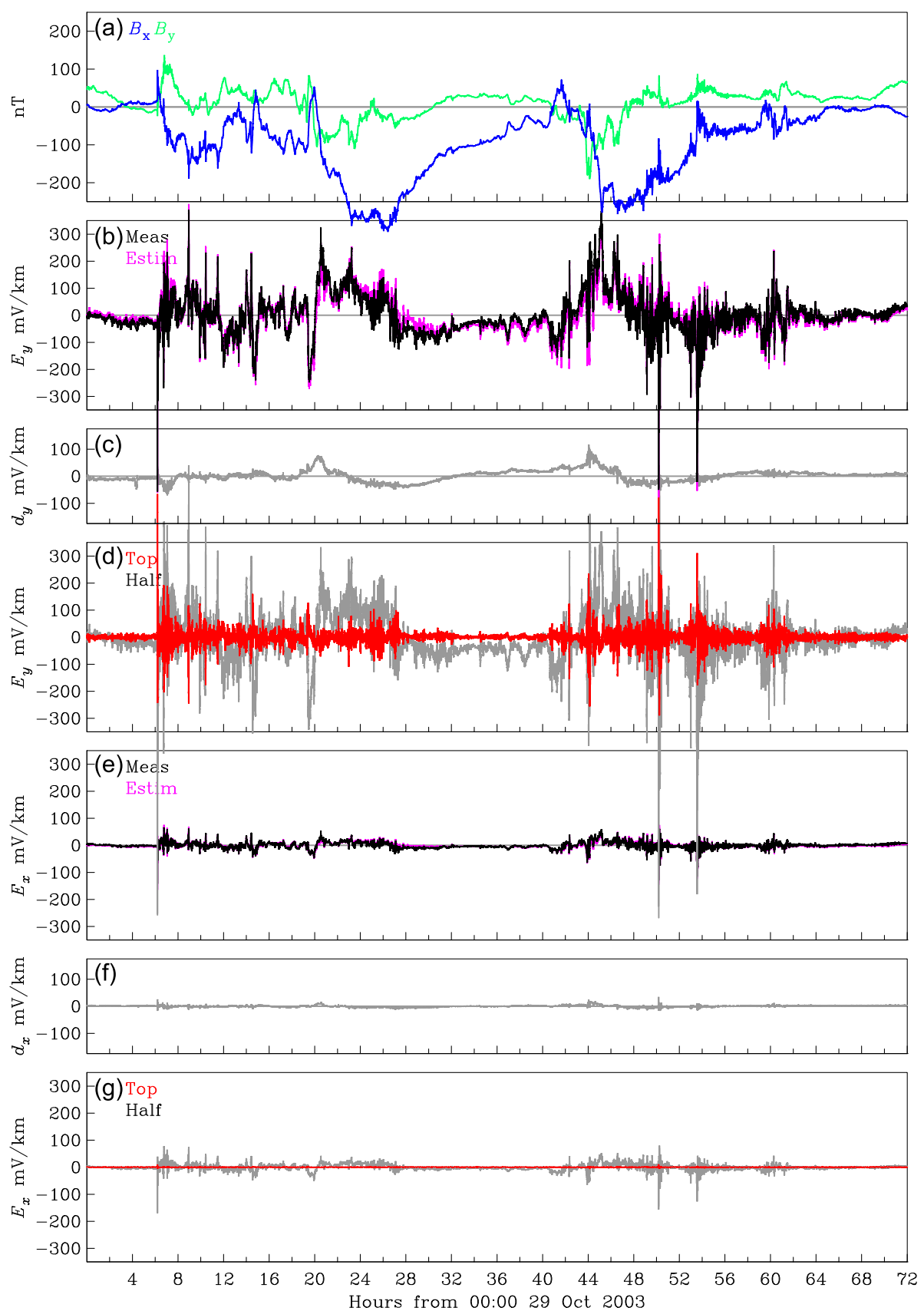

Figure 1 Time series recording the Halloween storm of 29 October - 31 October 2003, each with 1-s resolution. (a) Measured Kakioka geomagnetic variation relative to a quiet baseline, measured north $B_{x}^{m}$ (blue) and measured east $B_{y}^{m}$ (green); (b) east Kakioka geoelectric field variation, measured $E_{y}^{m}$ (black) and estimated $G E_{y}$ (purple); (c) residual difference $d_{y}$; (d) the parts of estimated $G E_{y}$ corresponding to induction in the top layer of the lithospheric model (red) and the lower half-space (gray); (e) north Kakioka geoelectric field variation, measured $E_{x}^{m}$ (black) and estimated $G E_{x}$ (purple); (f) residual difference $d_{x}$; and $(\mathbf{g})$ the parts of estimated $G E_{x}$ corresponding to induction in the top layer of the lithospheric model (red) and the lower half-space (gray).

This storm caused operational stress in the Swedish (Pulkkinen et al. 2005) and Scottish power grids (Thomson et al. 2005). It commenced with the arrival at the Earth of a coronal mass ejection, which abruptly compressed the magnetopause and generated a positive magnetic impulse in Kakioka $B_{x}^{m}$ of about $60 \mathrm{nT}$ at 06:13 UT on 29 October. Complex geomagnetic variation was driven by the arrival of additional coronal mass ejections, solar wind variability, and vigorous magnetospheric convection. This resulted in main-phase intensification 
of the ring current and a corresponding decrease in Kakioka $B_{x}^{m}$, with one minimum coming early in the day of 30 October and another a day later. The ring-current index reached a maximum value of $-D s t=383 \mathrm{nT}$ at 23:30 UT on 30 October. The maximum time-rate of change in the north component of the geomagnetic field $\left|\Delta B_{x}\right|$ during 29 to 31 October, calculated from first differences of the discrete 1-s data, was $5.58 \mathrm{nT} / \mathrm{s}$; the corresponding average $\left|\Delta B_{x}\right|$ was $0.07 \mathrm{nT} / \mathrm{s}$.

In Figure $1 \mathrm{~b}$,e we show, respectively, the $E_{y}^{m}$ east and $E_{x}^{m}$ north components of the geoelectric field measured at Kakioka for 29 to 31 October 2003 (black). We note that, in general, when the geomagnetic field is active, so is the geoelectric field, but the two data types are not simply correlated in time. We also note that $E_{y}^{m}$ has a much larger variational amplitude than $E_{x}^{m}$. This polarization is the results of geomagnetic induction in the three-dimensional conductivity distribution of the lithosphere beneath the observatory site. In the case of Kakioka, this apparently allows geoelectric fields to be more easily induced in the east direction than in the north direction.

Among the other data durations analyzed here, one records the Bastille-Day storm of 15 to 16 July 2000 (Yermolaev et al. 2008), which commenced with a sudden impulse of large amplitude and subsequently exhibited substantial geomagnetic variability; both maximum $\left|\Delta B_{x}\right|$ and average $\left|\Delta B_{x}\right|$ were comparable to the October 2003 Halloween storm. The storm of 20 to 21 November 2003 had a main-phase maximum -Dst that was greater than that of the Halloween storm but maximum and average $\left|\Delta B_{x}\right|$ that were less than those of the Halloween storm. The storm of 05 to 07 November 2001 included two abrupt geomagnetic impulses, a maximum $\left|\Delta B_{x}\right|$ greater than that of the Halloween storm, but an average $\left|\Delta B_{x}\right|$ less than that of the Halloween storm. In summary, the variety of geomagnetic time-dependencies exhibited by the five storms summarized in Table 1 allows us to test our algorithm for estimation of storm-time geoelectric fields.

\section{Transient induction for a flat Earth}

In seeking a practical method for estimating magnetic storm induction of electric fields in the lithosphere beneath a single ground-based observatory, it is natural to consider an idealized model having Cartesian geometry, where a unit positional vector is given by $\hat{x}=(\hat{x}, \hat{y}, \hat{z})$. The Earth's surface is represented by the plane $z=$ 0 ; the electrically conducting lithosphere, possibly with regional-scale three-dimensional structure, is assumed to occupy the region $(z>0)$; everything above the Earth's surface $(z<0)$ is assumed to be an insulator.

We consider geomagnetic field variation occurring over timescales of seconds to days and induction within the solid Earth over lithospheric depthscales of hundreds to thousands of kilometers. We can reasonably invoke the quasistatic approximation and neglect displacement currents in Maxwell's equations. The spatial form and temporal evolution of the geomagnetic field $\mathbf{B}$ is then given by the diffusion equation:

$$
\nabla^{2} \mathbf{B}-\mu \sigma \partial_{t} \mathbf{B}=0,
$$

where $t$ denotes time, $\sigma$ is the electrical conductivity (assumed, for now, to be spatially homogeneous), and $\mu$ is magnetic permeability. Magnetic time variation is related to an electric field $\mathbf{E}$ by Faraday's law:

$$
\nabla \times \mathbf{E}+\partial_{t} \mathbf{B}=0 .
$$

Following assumptions commonly made in magnetotelluric analyses, we invoke, as is often standard, the planewave approximation (Chave and Weidelt 2012), whereby geomagnetic and geoelectric field variations are assumed to lie in a horizontal plane $(\hat{x}, \hat{y})$ parallel to the surface of the Earth. With this, then, and as a consequence of Equation (1), we can assume that the geomagnetic field has the following separation of variables:

$$
\mathbf{B}(t, z)=\mathbf{B}_{s}(z)+\mathbf{B}_{-}(t) \mathrm{e}^{-k z}+\mathbf{B}_{+}(t) \mathrm{e}^{+k z},
$$

where $1 / k$ is the characteristic spatial depthscale and where $\mathbf{B}_{s}(z)$ is the secular part of the geomagnetic field that changes slowly in time compared to the duration of time considered here; we approximate it as time-steady, and we remark that its existence does not detectably affect our analysis. For convenience of plotting, we subtract a constant $\mathbf{B}(z)$ from the magnetic field data. Similarly, the geoelectric field has the form:

$$
\mathbf{E}(t, z)=\mathbf{E}_{-}(t) \mathrm{e}^{-k z}+\mathbf{E}_{+}(t) \mathrm{e}^{+k z},
$$

and we assume that this does not have a significant secular part.

Transient time signals are conveniently analyzed using the Laplace transform. A hypothetical signal $f(t)$ that varies over time and after some initial condition $(t \geq 0)$ is conceived as the superposition of exponential moments (e.g., Butkov 1968, Chs. 5 and 8]. The transformation of $f(t)$ into the complex frequency domain is given by:

$$
f(s)=\mathcal{L}\{f(t)\}=\int_{0}^{\infty} f(t) \mathrm{e}^{-s t} d t
$$

where $s$ is a complex frequency. Inverse transformation back to the time domain is given by

$$
f(t)=\mathcal{L}^{-1}\{f(s)\}=\frac{1}{2 \pi \mathrm{i}} \int_{\gamma-\mathrm{i} \infty}^{\gamma+\mathrm{i} \infty} f(s) \mathrm{e}^{s t} d s,
$$

where $\mathrm{i}=\sqrt{-1}$ and where integration is along a contour path in the complex $s$-plane with the positive real number $\gamma$ chosen so as to ensure convergence. Calculating an inverse Laplace transform using the Cauchy residue theorem often entails the discovery of a 'keyhole' contour path that circumvents singularities, but many inverse 
transformations, including that needed for this analysis, can be found in reference books. The Laplace transform of the time derivative of the function $f(t)$ is:

$$
\mathcal{L}\left\{\partial_{t} f(t)\right\}=s f(s)
$$

where we have assumed that the pre-response initial condition is assumed to be zero, $f(0)=0$.

The magnetic diffusion Equation (1) can be reduced to an algebraic equation by using the functional form (3) and the Laplace transform of the time derivative (7), giving:

$$
k^{2} \mathbf{B}-s \mu \sigma \mathbf{B}=0 .
$$

From this, we obtain the characteristic equation describing the relationship between the spatial wave number $k$ and the complex frequency $s$ :

$$
k=\sqrt{s \mu \sigma} \text {. }
$$

Next, recognizing that Faraday's Law (2) describes an orthogonal relationship between the inducing magnetic field $\mathbf{B}$ and the induced electric field $\mathbf{E}$, we use the characteristic Equation (9) to write:

$$
\mathbf{R E}(s, z)=C(s, z) \cdot s \mathbf{B}(s, z),
$$

where:

$$
\mathbf{R}=\left[\begin{array}{rr}
0 & -1 \\
1 & 0
\end{array}\right] \text { and } \mathbf{R}^{-1}=\left[\begin{array}{rr}
0 & 1 \\
-1 & 0
\end{array}\right]
$$

are spin matrices that come from the curl operator and are only two-dimensional because we have invoked the planewave approximation. $C(s, z)$ is the Schmucker-Weidelt (Schmucker 1973; Weidelt 1972) electromagnetic transfer function with impedance properties determined by the electrical conductivity of the lithosphere. $C(s, z)$ has units of length.

\section{Two-layer model}

In developing a model of lithospheric conductivity, we start simple, and then add details and model parameters that might be considered to be, at least, physically plausible. If the model leads to improved predictions of geoelectric fields at Kakioka, then the model can be considered to be 'useful.' We begin by recalling Cagniard's idealized model, which is often presented in textbooks on magnetotellurics: a one-dimensional lithosphere, consisting of a vertical stratification of $N$ horizontal layers, each having uniform conductivity (Cagniard 1953). We denote the conductivities from the top layer to the bottom as $\sigma_{n}=\sigma_{1}, \sigma_{2} \cdots \sigma_{N}$ and the layer thicknesses as $h_{n}=h_{1}, h_{2} \cdots h_{N}$, where the bottom $N$ th layer is assumed to be an infinite half-space, $h_{N}=\infty$. We denote the horizontal-layer interfaces by the sequence of depths $z_{n}=$ $z_{0}, z_{1} \cdots z_{N-1}$, where $z_{0}=0$ represents the surface of the Earth and $z_{N-1}$ is the top of the $N$ th layer. For this lithospheric geometry, Wait (1954) showed that, with recursive enforcement of continuity of magnetic and electric fields across each interface, the Schmucker-Weidelt $C$-functions can be expressed in terms of a recursion formula:

$$
C_{n}\left(s, z_{n-1}\right)=\frac{1}{k_{n}} \cdot \frac{k_{n} C_{n+1}\left(z_{n}\right)+\tanh \left(k_{n} h_{n}\right)}{1+k_{n} C_{n+1}\left(z_{n}\right) \tanh \left(k_{n} h_{n}\right)},
$$

where:

$$
C_{N}\left(s, z_{N-1}\right)=\frac{1}{k_{N}} \text {. }
$$

A model with just two horizontal layers is a reasonable incremental extension of the half-space model considered by Love and Swidinsky (2014). For this, the $C$-function at the Earth's surface reduces to:

$$
C_{1}\left(s, z_{0}\right)=\frac{1}{k_{1}} \cdot \frac{\left(k_{1} / k_{2}\right)+\tanh \left(k_{1} h_{1}\right)}{1+\left(k_{1} / k_{2}\right) \tanh \left(k_{1} h_{1}\right)} .
$$

This can be simplified even further if the top layer only moderately attenuates magnetic variation - that the electromagnetic skin depth of the top layer is much greater than its thickness (e.g., Simpson and Bahr 2005, p. 25). Indeed, near the Earth's surface, electrical conductivity can range over many orders of magnitude, but $\sigma_{1} \simeq$ $10^{-3} \mathrm{~S} / \mathrm{m}$ for the upper, say, $h_{1} \simeq 30 \mathrm{~km}$, would not be atypical. In this case, for a timescale of, say, $30 \mathrm{~s}$, the electromagnetic skin depth $1 / k_{1} \simeq 154 \mathrm{~km}$. With this, it is certainly plausible that $k_{1} h_{1} \simeq 0.19<<1$, in which case $\tanh \left(k_{1} h_{1}\right) \simeq k_{1} h_{1}$, and:

$$
C_{1}\left(s, z_{0}\right) \simeq \frac{1+k_{2} h_{1}}{k_{2}+k_{1}^{2} h_{1}} .
$$

Still more simplification can be obtained if the electromagnetic skin depth of the lower half-space is much greater than the thickness of the top layer. In the lower lithosphere, olivine has a conductivity of $\sigma_{2} \simeq 3 \times$ $10^{-4} \mathrm{~S} / \mathrm{m}$ (e.g., Yoshino and Katsura 2013). In this case, for a timescale of, say, $30 \mathrm{~s}$, the electromagnetic skin depth $1 / k_{2} \simeq 280 \mathrm{~km}$. In this case, $k_{2} h_{1} \simeq 0.11<<1$, and:

$$
C_{1}\left(s, z_{0}\right) \simeq \frac{1}{k_{2}+k_{1}^{2} h_{1}} .
$$

With this, we obtain the expression:

$$
C_{1}\left(s, z_{0}\right) \simeq \frac{1}{\mu \sigma_{1} h_{1}}\left(\frac{1}{s+\sqrt{a s}}\right) \equiv C_{0}(s),
$$

where we have defined, with simplified notation, the surface transfer function of interest $C_{0}(s)$. With these approximations, we identify a new timescale:

$$
\frac{1}{a}=\frac{\mu \sigma_{1}^{2} h_{1}^{2}}{\sigma_{2}} .
$$

Furthermore, we note that $C_{0}(s)$ is functionally dependent on the conductivity of the lower half-space layer $\sigma_{2}$ and the conductance of the top layer $\sigma_{1} h_{1}$; there is no separate functional dependence on the conductivity of the top layer $\sigma_{1}$ nor on its thickness $h_{1}$. 
We return to the time domain through inverse Laplace transformation (e.g., Abramowitz and Stegun 1965 eq. 29.3.43),

$$
\mathcal{L}^{-1}\left\{C_{0}(s)\right\}=C_{0}(t)=\frac{1}{\mu \sigma_{1} h_{1}} \exp (a t) \operatorname{erfc}(\sqrt{a t}),
$$

which is the impulse-response function (e.g., Kanasewich 1981 Ch. 5) relating the time derivative of the inducing geomagnetic field to the induced geoelectric field. But calculating the time derivative of a discrete time series is numerically unstable to even relatively small amounts of random noise. As a remedy, we choose to consider the impulse-response function for straight-line interpolation between successive discrete data values. For this, we need the Laplace transform of the corresponding 'ramp' function. This can be obtained by integrating Equation (19):

$$
C_{0}^{R}(t)=\int_{0}^{t} C_{0}(t-\tau) d \tau
$$

With this, we obtain the two-parameter impulse-response function:

$$
C_{0}^{R}(t ; a, b)=b \cdot\left[\exp (a t) \operatorname{erfc}(\sqrt{a t})+\frac{2}{\sqrt{\pi}} \sqrt{a t}\right],
$$

where:

$$
b=\frac{\sigma_{1} h_{1}}{\sigma_{2}}
$$

is a new depthscale.

We choose, now, to divide the response function into two parts:

$$
C_{0}^{R}(t ; a, b)=T_{0}^{R}(t ; a, b)+H_{0}^{R}\left(t ; a b^{2}\right) .
$$

The functional form of the first part:

$$
T_{0}^{R}(t ; a, b)=b \cdot \exp (a t) \operatorname{erfc}(\sqrt{a t}),
$$

is specified by two independent parameters, the frequency $a$ and the depthscale $b$, each of which depend, as we have noted, on the conductance of the top layer $\sigma_{1} h_{1}$ and conductivity of the lower half-space $\sigma_{2}$. On the other hand, the functional form of the second part of (23) is specified by just one parameter $a b^{2}$ :

$$
H_{0}^{R}\left(t ; a b^{2}\right)=\frac{2}{\sqrt{\pi}} \sqrt{a b^{2} t} .
$$

Since $a b^{2}=\left(\mu \sigma_{2}\right)^{-1}$, we note that this can be reexpressed as a function depending only on the conductivity $\sigma_{2}$ :

$$
H_{0}^{R}\left(t ; \sigma_{2}\right)=\frac{2}{\sqrt{\pi \mu \sigma_{2}}} \sqrt{t}
$$

which we note is the ramp response function for induction in a uniformly conducting lower half-space layer (e.g., Pirjola 1984, eq. 7), previously used by Love and Swidinsky
(2014) in their analysis of storm-time induction. From these observations, we recognize that induction within the top layer modifies induction within the lower half-space, but the total inductive effect is not simply an additive superposition: $T_{0}^{R}$ depends on both $\sigma_{1} h_{1}$ and $\sigma_{2}$, while $H_{0}^{R}$ only depends on $\sigma_{2}$. This asymmetry is a remnant of the derivation of $C_{0}(s)$, in which the equations of electromagnetism are solved through recursive matching of boundary conditions, starting from the bottom layer and then working up to the top layer.

\section{Application to discrete time series}

To apply these results to linear interpolation between discrete data values, we need the ramp response function corresponding to the sampling interval $\tau$ between each data value. We define this as:

$$
C_{\tau}^{R}(t) \equiv C_{0}^{R}(t) U(t)-C_{0}^{R}(t-\tau) U(t-\tau),
$$

where the Heaviside step function is:

$$
U(t-\tau)=\left\{\begin{array}{l}
0 \\
1
\end{array} \text { for } \begin{array}{r}
t<\tau \\
t>\tau
\end{array}\right.
$$

Next, we recognize that multiplication in the complex frequency domain corresponds to convolution in the time domain, so that for two functions $f$ and $g$ :

$$
\mathcal{L}^{-1}\{f(s) \cdot g(s)\}=(f * g)(t) .
$$

Therefore, discrete values of the induced electric field, denoted $\mathbf{E}\left(t_{i}\right)$, are obtained by discrete linear convolution with the ramp response function (27):

$$
\mathbf{R E}\left(t_{i} ; a, b\right)=\left(C_{\tau}^{R}(a, b) * \frac{\Delta \mathbf{B}}{\tau}\right)\left(t_{i}\right) .
$$

where:

$$
\Delta \mathbf{B}\left(t_{j}\right)=\mathbf{B}\left(t_{j+1}\right)-\mathbf{B}\left(t_{j}\right) .
$$

In detail, convolution is given by the integral:

$$
(f * g)(t)=\int_{0}^{t} f(t-\theta) \cdot g(\theta) d \theta .
$$

With this, Equation (30) can be written as:

$$
\mathbf{R E}\left(t_{i} ; a, b\right)=\sum_{j=1}^{i} C_{\tau}^{R}\left(t_{i}-t_{j} ; a, b\right) \cdot \frac{\Delta \mathbf{B}\left(t_{j}\right)}{\tau},
$$

which can be regarded as an applied form of Equation (10), suitable for algorithmic implementation.

\section{Parameterization for 3D conductivity}

Induction in the three-dimensional conductivity distribution of the lithosphere beneath an observatory site results in localized horizontal geoelectric fields having frequency-dependent polarization (e.g., Simpson and Bahr 2005, Ch. 5): Storm-time geoelectric fields will tend 
to be more easily induced in some azimuthal directions than in others, and the degree to which this is true will depend on the time rate of change of the geomagnetic field (e.g., McKay and Whaler 2006). Conversely, induction in a hypothetical half-space having uniform conductivity or, for that matter, in a Cartesian geometry with conductivity that is only depth dependent, with no lateral conductivity structure, will show no geoelectric polarization. Indeed, in a magnetotelluric analysis of data collected from a geographically distributed set of sensor systems, the frequency-dependent relationship between geomagnetic variation and polarized geoelectric variation is exploited in modeling three-dimensional lithospheric conductivity.

Residual polarization in the measured geoelectric field $\mathbf{E}^{m}$ not directly accommodated by the lithospheric conductivity model is treated as a 'galvanic' distortion of the modeled geoelectric field $\mathbf{E}$ :

$$
\mathbf{E}^{m}\left(t_{i}\right)=\mathbf{G E}\left(t_{i}\right)
$$

where the distortion tensor:

$$
\mathbf{G}=\left[\begin{array}{ll}
G_{x x} & G_{x y} \\
G_{y x} & G_{y y}
\end{array}\right]
$$

is assumed to be constant in time (frequency independent) (e.g., Groom and Bahr 1992; Jones 2012). Galvanic distortion is often regarded as a nuisance for magnetotelluric analysis. It is sometimes attributed to the quasi-static accumulation of electric charges along the boundaries of spatial heterogeneities in lithospheric conductivity and within conductivity gradients. In the context of inductionhazard science and the need to develop algorithms for estimating storm-time induction of geoelectric fields, lithospheric conductivity is often modeled as being only depth dependent (e.g., Pulkkinen et al. 2007). Recently, however, three-dimensional models have been used in the analysis of storm-time induction (e.g., Püthe and Kuvshinov 2013; Thomson et al. 2009; Viljanen et al. (2014); in their independent analyses of Kakioka geomagnetic and geoelectric data, Love and Swidinsky Love and Swidinsky (2014) and Püthe et al. 2014 each used a time-constant galvanic distortion tensor in their modeling of the polarizing effects of localized three-dimensional lithospheric conductivity.

Given our practical goal of obtaining improved estimates of storm-time geoelectric fields, we choose to extend the parameterizations of the preceding analyses in two different ways. First, we recognize that the development in the 'Two-layer model' section is an idealization, the details of which do not need to be slavishly retained. We choose to remove the parametric interdependence of the top $T_{0}^{R}$ and half-space $H_{0}^{R}$ response functions; instead of two independent parameters $\{a, b\}$, we allow for three $\left\{a_{T}, b_{T}, \sigma_{H}\right\}$, and instead of (23), we have:

$$
C_{0}^{R}\left(t ; a_{T}, b_{T}, \sigma_{H}\right)=T_{0}^{R}\left(t ; a_{T}, b_{T}\right)+H_{0}^{R}\left(t ; \sigma_{H}\right) .
$$

With this, we can calculate independent top and halfspace model electric fields:

$$
\mathbf{E}\left(t_{i} ; a_{T}, b_{T}, \sigma_{H}\right)=\mathbf{E}_{T}\left(t_{i} ; a_{T}, b_{T}\right)+\mathbf{E}_{H}\left(t_{i} ; \sigma_{H}\right) .
$$

where, from Equation (30):

$$
\mathbf{R E}_{T}\left(t_{i} ; a_{T}, b_{T}\right)=T_{\tau}^{R}\left(a_{T}, b_{T}\right) * \frac{\Delta \mathbf{B}}{\tau}
$$

and:

$$
\mathbf{R E}_{H}\left(t_{i} ; \sigma_{H}\right)=H_{\tau}^{R}\left(\sigma_{H}\right) * \frac{\Delta \mathbf{B}}{\tau} .
$$

Second, we choose to assign independent distortion tensors to the model top and half-space electric fields:

$$
\mathbf{E}^{m}\left(t_{i}\right)=\mathbf{G}_{T} \mathbf{E}_{T}\left(t_{i}\right)+\mathbf{G}_{H} \mathbf{E}_{H}\left(t_{i}\right) .
$$

To avoid parametric redundancy, we require that each of the distortion matrices satisfy the Frobenius norm:

$$
\operatorname{Tr}\left(\mathbf{G G}^{T}\right)=2 .
$$

With this, the independent model parameters, denoted $\left\{a_{T}, b_{T}, \sigma_{H}, \mathbf{G}\right\}$, are nine in number.

Since the response function for the top layer (24) has a time-functional form that is different from that of the half-space (26), by assigning separate distortion tensors to each of the top and half-space transfer functions, we are allowing geoelectric polarization to have frequency dependence, such as can arise from induction in a lithosphere having three-dimensional conductivity. This generalization permits improved fits of the model-estimated geoelectric fields to the measured (and, generally, polarized) geoelectric fields, which is our objective. If this also partly obscures the physical interpretation that might be made of the electrical conductivity properties of the top and half-space model lithospheric layers, this can be accepted so long as the same model parameters provide reasonably accurate estimates of induced geoelectric fields for different magnetic storms.

\section{Parameter estimation}

We define the residual discrepancy between the measured geoelectric field and that which we estimate from induction:

$$
\mathbf{d}\left(a_{T}, b_{T}, \sigma_{H}, \mathbf{G}\right)=\mathbf{E}^{m}-\mathbf{G}_{T} \mathbf{E}_{T}-\mathbf{G}_{H} \mathbf{E}_{H},
$$

Using the Kakioka magnetic and electric field data for each storm, summarized in the 'Observatory data' section, we obtain a set of model parameters by minimization of the squared misfit:

$$
\varepsilon^{2}=\sum_{i} \mathbf{d}\left(t_{i}\right) \cdot \mathbf{d}\left(t_{i}\right) / \sum_{i} \mathbf{E}^{m}\left(t_{i}\right) \cdot \mathbf{E}^{m}\left(t_{i}\right),
$$


which we obtain with a Powell algorithm (Press et al. 1992, Ch. 10.5). We require $a=|a|$, and for numerical stability in calculating Equation (21), we first calculate $a t+$ $\log [\operatorname{erfc}(\sqrt{a t})]$ and then take its exponential. Given that the number of 1-s data values for a 3-day duration of time is large, 518,400 , it is useful to design an efficient computer algorithm for minimizing $\varepsilon^{2}$. We define data-fitting efficiency, or 'variance reduction,' as $1-\varepsilon^{2}$.

\section{Results}

In Table 1, we summarize lithospheric model parameters $\left\{a_{T}, b_{T}, \sigma_{H}, \mathbf{G}\right\}$ and data misfits determined, separately, for each of the five storms. In Figure 1b,e, we compare, respectively east and north, components of the measured geoelectric field ( $\mathbf{E}^{m}$, black) at Kakioka against the estimated geoelectric field (GE, purple) - purple variation approximately equals black variation. Optimal parameters (\#4 in Table 1) for the two-layer model leave a misfit residual of only $\varepsilon^{2}=0.0686$; this means that the model accounts for $93 \%$ of the variance in the 2003 Halloween storm 1-s Kakioka $\mathbf{E}^{m}$ data. These results are an improvement over those of Love and Swidinsky (2014), whose minimal half-space model accounted for $87 \%$ of the variance in the Kakioka electric field data for the Halloween storm but where high-frequency variation was not as well estimated. Still, even with the two-layer model presented here, some small residual signal remains, much of which varies over an approximate diurnal timescale. This can be seen in Figure 1c,f, where we plot the residual differences, Equation (42), between the measured and estimated geoelectric fields. The roles played, respectively, by the top and half-space lithospheric layers in affecting the estimated geoelectric field $\mathbf{E}$ can be seen in Figure 1d,g, where we plot the top $\mathbf{G}_{T} \mathbf{E}_{T}$ (red) and the half-space $\mathbf{G}_{H} \mathbf{E}_{H}$ (gray) time series. Note that the top layer mostly supports high-frequency electric field variation, while the half-space layer supports variation across a broad range of frequencies.

Geoelectric variation at Kakioka is even more accurately estimated for the other four storms. Still, the optimal model parameters $\left\{a_{T}, b_{T}, \sigma_{H}, \mathbf{G}\right\}$ show what might seem to be substantial variance between estimates for different storms, especially for the model top layer. Since the conductance of the top layer allows modeling of highfrequency electric field variation, the relative amount of such variation among the five different storms ends up determining a relatively different set of parameters for the top layer. We seek to identify a more stable set of model parameters: the average values of $\left\{a_{T}, b_{T}, \sigma_{H}, \mathbf{G}\right\}$ are listed in Table 1 and labeled '\#A.' We also list residual misfits $\varepsilon^{2}$ for the average parameters for each of the five storms; in each case, the misfits are only slightly greater than the fits obtained by optimal parameters determined separately for each storm; for example, the \#A parameters give a residual misfit of $\varepsilon^{2}=0.0816$ for the Halloween storm. In more detail, the degree to which the estimated geoelectric fields successfully match those measured at Kakioka can be seen in Figure 2, where we show just 20 min of 1-s $E_{y}$ variation before and after the commencement of the Halloween storm. Given the complexity of the time series, and the relative simplicity of our model (just nine parameters), it is satisfying that we can estimate geoelectric variation with a high degree of accuracy.

\section{Interpretation of model lithosphere}

Since our analysis here is concerned with the development and testing of an algorithm for estimating the storm-time induction of geoelectric fields, assumptions about the lithosphere and specific model parameterizations can be viewed in purely practical terms - they enable estimation. Detailed inference about the actual nature of the Earth's lithosphere is not, itself, a goal. At the same time, we recognize that a realistic model of the lithosphere should, in principle, facilitate accurate geoelectric estimation, and so, it is reasonable to compare our simple model with what is either known or independently inferred about the lithosphere.

As noted in the previous section, rapid geomagnetic induction is supported by the conductance of the top layer of the model lithosphere. For the parameters of the average lithospheric model A, the diffusive timescale of the top layer is:

$$
\frac{1}{a_{T}}=24.08 \mathrm{~s}
$$

which is relatively short, on the order of half the rise time of a magnetic impulse typically seen during an intense storm. The corresponding depthscale and normalized distortion tensor are:

$$
b_{T}=47.50 \mathrm{~km}, \quad \mathbf{G}_{T}=\left[\begin{array}{cc}
-0.03 & 0.02 \\
-0.70 & 1.23
\end{array}\right],
$$

while the underlying half-space part of the model has the conductivity parameters:

$$
\sigma_{H}=3.5 \times 10^{-4} \mathrm{~S} / \mathrm{m}, \quad \mathbf{G}_{H}=\left[\begin{array}{rr}
0.06 & 0.18 \\
-0.28 & 1.37
\end{array}\right] .
$$

Of course, the Japanese archipelago and its regional surroundings are geologically and geophysically complicated. And, indeed, published magnetic sounding and magnetotelluric analyses of the lithosphere beneath and around Honshu (Fuji-ta et al. 1997; Kasaya et al. 2005; Ogawa et al. 1986), the island on which the Kakioka observatory is situated, reveal a complicated three-dimensional conductivity distribution: near the surface, sea water is a very good electrical conductor, $3 \mathrm{~S} / \mathrm{m}$; crustal conductivity ranges from about $10^{-2}$ to $10^{-1} \mathrm{~S} / \mathrm{m}$; below this, and across lithosphere depths down to about $30 \mathrm{~km}$, the electrical conductivity is estimated to be relatively 


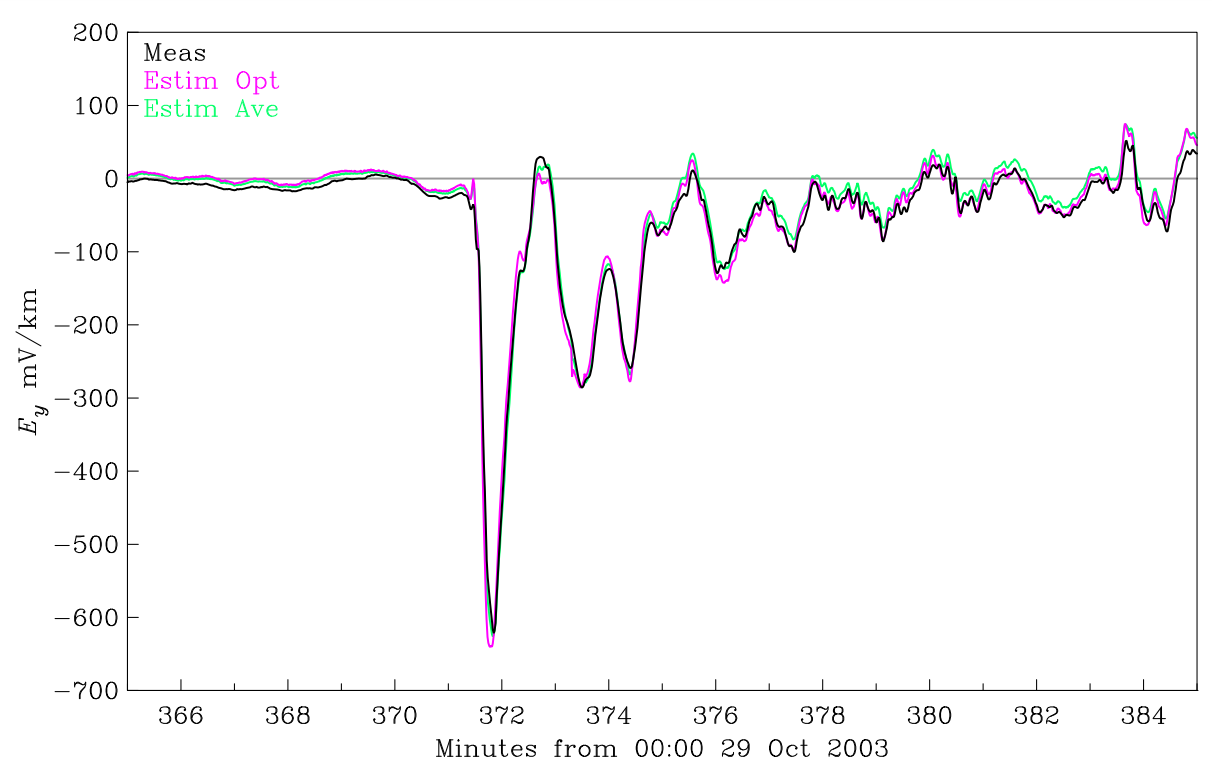

Figure 2 Time series recording 20 min of east geoelectric field variation in the early stage of the 2003 Halloween storm. Shown are measured $E_{y}^{m}$ data (black), estimated $G E_{y}$ variation for optimal model parameters determined from Kakioka data recording the 2003 Halloween storm (\#4 in Table 1, purple), and estimated $G E_{y}$ variation for model parameters determined from five different magnetic storms (\#A in Table 1, green).

low, $10^{-4} \mathrm{~S} / \mathrm{m}$, but there are possibly isolated parts with dimensions of $10 \mathrm{~km}$ or so having conductivity of $10^{-1} \mathrm{~S} / \mathrm{m}$; deeper still, and down to depths greater than $100 \mathrm{~km}$, conductivity ranges from $10^{-2}$ to $10^{-4} \mathrm{~S} / \mathrm{m}$, with lower conductivity structures possibly corresponding to the subducting Pacific and the overriding Okhotsk plates. The complicated regional distribution of electrical conductivity is almost certainly responsible for the high degree of polarization in the induced geoelectric field measured at Kakioka.

Püthe et al. 2014 have noted a seeming inconsistency between their estimate of the Kakioka galvanic distortion tensor, obtained for a spherical model, and that reported by Love and Swidinsky (2014, eq. 29), obtained for a Cartesian half-space. Püthe et al. speculate that this might be an artifact of the modeling method used by Love and Swidinsky. In fact, the explanation is much more mundane than that; Love and Swidinsky simply made a mistake in their reporting of the tensor elements: unintended rotation of the distortion tensor. Other results reported by Love and Swidinsky, including the average inferred conductivity and fits to the electric field data, are unaffected by this mistake. The elements reported here have been checked carefully for placement and sign; given expected statistical jitter, the half-space elements for $\mathbf{G}_{H}$ are certainly reasonably consistent with those estimated by Püthe et al. (2014).

\section{Conclusions and new questions}

The preceding work demonstrates the opportunity potential for algorithmic estimation of geoelectric fields induced in the Earth's electrically conducting lithosphere during magnetic storms. It also illuminates the possibility that real-time algorithms for geoelectric estimation can be developed using a Laplace-transform formalism, thus ensuring time-causal dependence on (only) past geomagnetic field variation. Still, we can also see some challenges for induction-hazard science. The polarization of geoelectric data recorded at Kakioka requires parameterization for three-dimensional lithospheric conductivity, which we accomplish here, in terms of distortion tensors. From this, we can understand that mapping of hazardous geoelctric fields across the Earth's surface will require detailed modeling of the three-dimensional lithospheric conductivity. Furthermore, by virtue of localized differences in lithospheric conductivity, storm-time geoelectric variation realized at one observatory site will not always be well correlated with geoelectric variation at another site. It would be of interest to study the correlation between pairs of geoelectric time series and how it falls off as a function of increasing site separation. This could be accomplished by deploying, on a temporary basis, measurement systems separated by different distances and then waiting for an intense magnetic storm to occur. Another issue that remains insufficiently studied is storm-time induction at high auroral-zone latitudes, where geomagnetic activity can have amplitudes much greater than at mid and low latitudes. Unfortunately, very few detailed geoelectric time series are available from auroral latitudes. In light of these limitations, we believe that a modest expansion of long-term geoelectric monitoring would be beneficial for induction-hazard science. 


\section{Competing interests}

The authors declare that they have no competing interests.

\section{Authors' contributions}

The basic problem addressed in this paper came out of conversations between JJL and AS. JJL developed the mathematics in consultation with AS $J \mathrm{JL}$ wrote the computer programs and performed the data analysis. JJL wrote the first draft of a manuscript, while AS provided important input for content. Both authors read and approved the final manuscript.

\section{Acknowledgements}

We thank CA Finn, EJ Rigler, JL Slate, and two anonymous people for reviewing a draft manuscript. We thank PA Bedrosian and A Kelbert for useful conversations.This analysis could not have been done without the high-quality and high-continuity geomagnetic and geoelectric field data acquired by the JMA at the Kakioka observatory. We thank Püthe et al. for their attentive examination of our previously published results Love and Swidinsky 2014. This work was supported by the USGS Geomagnetism Program.

\section{Author details}

${ }^{1}$ US Geological Survey, Geomagnetism Program, Box 25046 MS 966 DFC, Denver, CO 80225, USA. ²Department of Geophysics, Colorado School of Mines, Golden, CO 80401, USA.

\section{Received: 23 August 2014 Accepted: 14 March 2015}

\section{Published online: 28 April 2015}

\section{References}

Abramowitz M, Stegun IA (1965) Handbook of mathematical functions. Dover, New York, NY. 1-1046

Allen J, Frank L, Sauer H, Reiff P (1989) Effects of the March 1989 solar activity. Eos Trans Am Geophys Union 70:1479-14861488

Baker DN, Balstad R, Bodeau JM, Cameron E, Fennell JE, Fisher GM, Forbes KF, Kintner PM, Leffler LG, Lewis WS, Reagan JB, Small AA, Stansell TA, Strachan $L$ (2008) Severe space weather events - understanding societal and economic impacts. The National Academy Press, Washington, DC. pp 1-144

Balch C, Murtagh B, Zezula D, Combs L, Nelson G, Tegnell K, Crown M, McGehan B (2004) Service assessment: intense space weather storms October 19 November 07 2003. US Dept Commerce, NOAA, Silver Spring, MD. pp 1-49

Bolduc L (2002) GIC observations and studies in the Hydro-Québec power system. J Atmos Solar-Terr Phys 64:1793-1802

Boteler DH, Pirjola RJ, Nevanlinna H (1998) The effects of geomagnetic disturbances on electrical systems at the Earth's surface. Adv Space Res 22:17-27

Butkov E (1968) Mathematical physics. Addison-Wesley, New York, NY. pp $1-735$

Cagniard L (1953) Basic theory of the magneto-telluric method of geophysical prospecting. Geophysics 18:605-635

Cannon P, Barclay L, Curry C, Dyer C, Edwads R, Greene G, Hapgood M, Horne RB, Jackson D, Mitchell CN, Owen J, Richards A, Rogers C, Ryden K, Saunders S, Sweeting M, Tanner R, Thomson A, Underwood C (2013) Extreme space weather: impacts on engineered systems and infrastructure. Roy Acad Engineer, London, UK. pp 1-68

Chave AD, Jones AG (eds) (2012) The magnetotelluric method. Cambridge Univ Press, Cambridge, UK. pp 1-552

Chave AD, Weidelt P (2012) The theoretical basis for electromagnetic induction. In: Chave AD, Jones AG (eds). The magnetotelluric method. Cambridge Univ Press, Cambridge, UK. pp 19-49

Daglis IA (ed) (2004) Effects of space weather on technology infrastructure. Kluwer Academic Publishers, Dordrecht, The Netherlands. pp 1-334

Ferguson IJ (2012) Instrumentation and field procedure. In: Chave AD, Jones AG (eds). The magnetotelluric method. Cambridge Univ Press, Cambridge, UK. pp 421-479

Fuji-ta K, Ogawa Y, Yamaguchi S, Yaskawa K (1997) Magnetotelluric imaging of the SW Japan forearc - a lost paleoland revealed? Phys Earth Planet Int 102:231-238

Gopalswamy N, Barbieri L, Cliver EW, Lu G, Plunkett SP, Skoug RM (2005) Introduction to violent Sun-Earth connection events of October-November 2003. J Geophys Res 110. A09S00, doi:10.1029/2005JA011268
Groom RW, Bahr K (1992) Corrections for near surface effects: decomposition of the magnetotelluric impedance tensor and scaling corrections for regional resistivities: a tutorial. Surv Geophys 13:341-379

Jones AG (2012) Distortion of magnetotelluric data: its identification and removal. In: Chave AD, Jones AG (eds). The Magnetotelluric Method. Cambridge Univ Press, Cambridge, UK. pp 219-302

Kanasewich ER, Univ Alberta Press, Alberta Canada (1981) Time sequence analysis in geophysics. pp 1-480

Kappenman JG (2012) A perfect storm of planetary proportions. IEEE Spectrum 49:26-31

Kasaya T, Goto T, Mikada H, Baba K, Suyehiro K, Utada H (2005) Resistivity image of the Philippine Sea plate around the 1944 Tonankai earthquake zone deduced by marine and land MT surveys. Earth Planets Space 57:209-213

Lloyds (2013) Emerging risk report: solar storm risk to the North American electric grid. Lloyd's of London, London, UK. pp 1-22

Love JJ (2008) Magnetic monitoring of Earth and space. Physics Today 61(2):31-37

Love JJ, Chulliat A (2013) An international network of magnetic observatories. Eos Trans Am Geophys Union 94(42):373-384. doi:10.1002/2013EO42000

Love JJ, Finn CA (2011) The USGS Geomagnetism Program and its role in space weather monitoring. Space Weather 9. S07001, doi:10.1029/2011SW000684

Love JJ, Swidinsky A (2014) Time causal operational estimation of electric fields induced in the Earth's lithosphere during magnetic storms. Geophys Res Lett 41:2266-2274. doi:10.1002/2014GL059568

Love JJ, Rigler EJ, Pulkkinen A, Balch CC (2014) Magnetic storms and induction hazards. Eos Trans Am Geophys Union 95(48):445-446. doi:10.1002/2014EO480001

Macmillan S (2007) Observatories, overview. In: Gubbins D, Herrero-Bervera E (eds). Encyclopedia of geomagnetism and paleomagnetism. Springer, New York, NY. pp 708-711

Minamoto Y (2013) Availability and access to data from Kakioka Magnetic Observatory, Japan. Data Sci J 12:30-35

McKay AJ, Whaler KA (2006) The electric field in northern England and southern Scotland implications for geomagnetically induced currents. Geophys J Int 167:613-625. doi:10.1111/j.1365-246X.2006.03128.x

Ogawa Y, Yukutake T, Utada H (1986) Two-dimensional modelling of resistivity structure beneath the Tohoku District, Northern Honshu of Japan, by a finite element method. J Geomag Geoelectr 38:45-79

Pirjola R (1984) Estimation of the electric field on the Earth's surface during a geomagnetic variation. Geophysica 20:89-103

Press WH, Teukolsky SA, Vetterling WT, Flannery BP (1992) Numerical Recipes. Cambridge Univ Press, Cambridge, UK, pp 1-963

Pulkkinen A, Lindahl S, Viljanen A, Pirjola R (2005) Geomagnetic storm of 29-31 October 2003: Geomagnetically induced currents and their relation to problems in the Swedish high-voltage power transmission system. Space Weather 3 S08C03. doi:10.1029/2004SW000123

Pulkkinen A, Pirjola R, Viljanen A (2007) Determination of ground conductivity and system parameters for optimal modeling of geomagnetically induced current flow in technological systems. Earth Planets Space 59:999-1006

Pulkkinen A, Bernabeu E, Eichner J, Beggan C, Thomson AWP (2012) Generation of 100-year geomagnetically induced current scenarios. Space Weather 10 S04003. doi:10.1029/2011SW000750

Püthe C, Kuvshinov A (2013) Towards quantitative assessment of the hazard from space weather Global 3-D modellings of the electric field induced by a realistic geomagnetic storm. Earth Planets Space 65:1017-1025

Püthe C, Manoj C, Kuvshinov A (2014) Reproducing electric field observations during magnetic storms by means of rigorous 3-D modelling and distortion matrix co-estimation. Earth Planets Space 66:162 doi:10.1186/s40623-014-0162-2

Riswadkar AV, Dobbins B (2010) Solar storms: protecting your operations against the Sun's 'Dark Side'. Zurich Services Corp, Schaumburg, pp 1-12

Schultz A (2009) A continental scale magnetotelluric observatory and data discovery resource. Data Sci J 8:6-20

Schmucker U (1973) Regional induction studies: a review of methods and results. Phys Earth Planet Inter 7:365-378

Simpson F, Bahr K (2005) Practical magnetotellurics. Cambridge Univ Press, Cambridge, UK. 1-254

Thomson AWP, McKay AJ, Clarke E, Reay SJ (2005) Surface electric fields and geomagnetically induced currents in the Scottish power grid during the 30 October 2003 geomagnetic storm. Space Weather 3. S11002, doi:10.1029/2005SW000156 
Thomson AWP, McKay AJ, Viljanen A (2009) A review of progress in modelling of induced geoelectric and geomagnetic fields with special regard to induced currents. Acta Geophys 57:209-219

Thomson AWP, Gaunt CT, Cillers P, Wild JA, Opperman B, McKinnell LA, Kotze P, Ngwira CM, Lotz SI (2010) Present day challenges in understanding the geomagnetic hazard to national power grids. Adv Space Res 45:1182-1190

Uyeshima M (2007) EM monitoring of crustal processes including the use of the network-MT observations. Surv Geophys 28:199-237. doi:10.1007/s10712-007-9023-x

Viljanen A (1997) The relation between geomagnetic variations and their time derivatives and implications for estimation of induction risk. Geophys Res Lett 24:631-634

Viljanen A, Pirjola R, Prácser E, Katkalov J, Wik M (2014) Geomagnetically induced currents in Europe: modelled occurrence in a continent-wide power grid. J Space Weather Space Clim 4(A09). doi:10.1051/swsc/2014006

Wait JR (1954) On the relation between telluric currents and the Earth's magnetic field. Geophysics 19:281-289

Weidelt P (1972) The inverse problem of geomagnetic induction. Z Geophys 38:257-289

Wei LH, Homeier N, Gannon JL (2013) Surface electric fields for North America during historical geomagnetic storms. Space Weather 11:451-462. doi:10.1002/swe.2007

Yermolaev YI, Zelenyi LM, Kuznetsov VC, Chertok IM, Panasyuk MI, Myagkova IN, Zhitnik IA, Kuzin SV, Exelevich VG, Bogod VM, Arkhangelskaja IV, Arkhangelsky Al, Kotov YD (2008) Magnetic storm of November, 2004 solar, interplanetary, and magnetospheric disturbances. J Atmos Solar-Terr Phys 70:334-341

Yoshino T, Katsura T (2013) Electrical conductivity of mantle minerals: role of water in conductivity anomalies. Ann Rev Earth Planet Sci 41:605-628

\section{Submit your manuscript to a SpringerOpen ${ }^{\circ}$ journal and benefit from:}

- Convenient online submission

- Rigorous peer review

- Immediate publication on acceptance

- Open access: articles freely available online

- High visibility within the field

- Retaining the copyright to your article

Submit your next manuscript at $\gg$ springeropen.com 